\title{
Field representation for optical defect resonances in multilayer microcavities using quasi-normal modes
}

\author{
M. Maksimovic, M. Hammer, E. van Groesen \\ MESA+ Institute for Nanotechnology, University of Twente, The Netherlands
}

\begin{abstract}
Quasi-Normal Modes are used to characterize transmission resonances in 1D optical defect cavities and the related field approximations. We specialize to resonances inside the bandgap of the periodic multilayer mirrors that enclose the defect cavities. Using a template with the most relevant QNMs a variational principle permits to represent the field and the spectral transmission close to resonances.
\end{abstract}

Key words: photonic bandgap, cavity resonance, eigenfrequency, quasi-normal mode, multilayer

PACS: 42.25.Bs, 42.70.Qs, 42.82.-m

\section{Introduction}

When subjected to external excitation, periodic multilayer structures show a resonant response in the time or frequency domain, which can be tailored by inclusion of defects [1-4]. Finite structures can be viewed as open systems which permit the leakage of energy to the exterior, described by the Helmholtz equation with outgoing wave boundary conditions. This constitutes an eigenvalue problem for complex frequencies and the associated field profiles, or quasi-normal modes (QNMs) [5].The quasi-normal modes specify the field patterns in which the leaky optical structure would oscillate naturally after an initial excitation is withdrawn, representing damped oscillatory solutions of the wave equation [6,21]. QNMs and associated complex eigenvalues can be viewed as a proper model for solving the problem of energy leaking out of open structures, see [2,5-7] and references therein.

Our aim is to use the characterization of the optical microcavity structures in terms of quasi-normal modes to describe approximately the resonant response to external excitation in the frequency domain and the related field profiles. 
For specific configurations the complex QNM eigenvalues appear to correspond to the position and quality of resonances in the spectral transmission. Although this holds when spectral resonances are sufficiently far apart from each other, this is not a general property and real frequencies of transmission resonances can be quite different form the real parts of the complex QNM eigenfrequencies in the given frequency range [8].

Field representations using QNMs have been investigated in [8-12], on the basis of quasi-normal mode theory as established in [6], founded on certain completeness properties and a linear-space structure for QNMs [13-15]. Orthogonality of QNMs is expressed with a specific bilinear form that includes boundary terms and in contrast to the usual inner product does not define a real, positive definite norm $[16,17]$. An eigenfunction expansion based on this bilinear form [6,20], used as a means for projecting functions onto the QNM basis, can furnish a field representation only over a finite spatial domain (due to exponentially growing envelopes of QNM basis functions) and under certain conditions necessary for completeness, as detailed in [13]. The completeness properties of QNMs have been addressed also in $[18,19]$.

When applying a QNM expansion method to transmission problems, several points are important. First, individual QNMs do not satisfy the proper boundary conditions for the transmittance problem directly. The incoming wave contribution in the transmission problem is introduced via time dynamic equations for the expansion coefficients. Frequency domain equations are obtained by Fourier transform [11], [20]. Second, as detailed in reference [20], an expansion based solely on QNMs can represent the internal cavity field up to the boundary of the enclosed region, with exception of a set of measure zero (the boundary points). This means that QNM expansions permit convergence in the mean but not pointwise. This situation arises specifically when the relevant field does not satisfy the same outgoing wave boundary conditions as the QNMs. Hence, despite the completeness property, in these cases finite, truncated QNM expansions lead to poorly converging field representations. Only after applying certain summation rules following from the completeness relations $[6,20]$ a better convergence might be achieved. Still, adequate approximations of the fields usually require summations over many basis modes (although this hardly ever seems to have been observed explicitly, perhaps due to comparisons of intensity shapes in place of field profiles). In addition some caution is necessary when taking into account contributions of a single resonance in the field representation based on QNM expansion, as emphasized in the reference [20]. This holds even in a spectral region of isolated, defect-induced transmission resonances in the bandgap, where one would expect that only those QNMs with the real parts of their eigenfrequencies in this frequency region are sufficient.

Alternative approaches in describing leaky optical structures are reported in literature and field representations in open 1D cavity structures are considered, see [21] and references therein. These methods primarily consider quantum theory of open systems and do not establish a direct connection between transmittance (scattering) 
problems and QNMs for general structure.

As far as we are aware no adequate generalizations to 2D and 3D structures are reported in literature of the above mentioned approaches. This is also true for PBG (photonic bandgap) structures that we are primarily interested in. Some attempts to use a QNM-like (eigenmode) description for 2D and 3D PBG structures are those related to a scattering-matrix approach as reported in [22,23] and [24].

It is the purpose of this paper to establish a quantitative relation between the description of the structure under external excitation with given fixed frequency (the transmittance problem) and the eigenvalue problem for QNMs, emphasizing the nature of realistic open structures. Our method specializes to optical defect structures where high-Q resonances are present inside the photonic bandgap. As detailed in this paper, it turns out that the variational form of the transmission problem offers a resourceful alternative to existing methods when applied to description of the fields and transmission responses of the localized defect modes formed inside the photonic bandgap. Our method does not rely on any completeness properties of QNMs nor on a bilinear form for projecting fields onto the QNM basis.

The approach proposed in section 2 uses a combination of the bandgap field of the structure (without defect) and only one/few relevant QNM(s) as a template. By restricting a specific functional one obtains approximations for the spectral power transfer and the optical field related to the transmission through the defect structure. In section 3 we analyze single and multiple defect cavities in finite 1D periodic structures for both symmetric and non-symmetric layer arrangements. Real world structures are bound to be finite and this feature is explicitly incorporated by the present approach, in contrast to techniques that rely on artificial periodization in Bloch-type analysis and supercell methods that can introduce nonphysical and spurious solutions, although usefulness and applicability of these methods is proven and well established in practice $[2,3]$.

\section{Theory}

We consider problems in 1D for structures with arbitrary piecewise constant refractive index distribution $n(x)$ within a finite domain $x \in(0, L)$ and assume that the structure is enclosed by two semi infinite domains with constant refractive indices $n_{\text {in }}, n_{\text {out }}$ as depicted in Fig. 1 .

We choose a harmonic time dependence for the electric field

$$
E(x, t)=E(x) e^{-i \omega t} .
$$

Therefore, the response of the structure under external excitation is described by the Helmholtz equation 


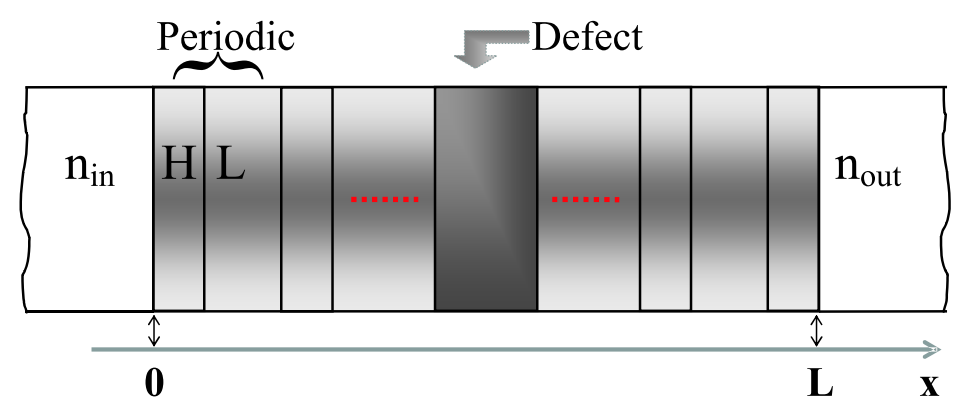

Fig. 1. The (defect) grating is a finite periodic structure consisting of two materials with high index $n_{H}$ and low index $n_{L}$. The layer thicknesses $L_{H}, L_{L}$ are quarter-wavelength for the target wavelength. Optical defects are introduced as changes of layer thicknesses. The grating is surrounded by two semi-infinite media of indices $n_{\text {in }}$ and $n_{\text {out }}$.

$$
\partial_{x}^{2} E(x)+k^{2}(x) E(x)=0
$$

with a transparent influx boundary condition at the side of the structure where the incident wave $\left(E_{i n c}=A_{i n c} e^{i k_{i n} x}\right)$ impinges, and a transparent boundary condition at the other side

$$
\begin{gathered}
\left.\left(\partial_{x}+i k_{\text {in }}\right) E\right|_{x=0}=2 i k_{i n} A_{\text {inc }}, \\
\left.\left(\partial_{x}-i k_{\text {out }}\right) E\right|_{x=L}=0,
\end{gathered}
$$

where $k(x)=\omega^{2} n^{2}(x) / c^{2}, k_{\text {in }}=n_{\text {in }} \omega / c$ and $k_{\text {out }}=n_{\text {out }} \omega / c$, for given amplitude of the incident wave $A_{i n c}$ and frequency $\omega$. This is the transmittance problem, where the field distribution inside and outside the structure for given real frequency $\omega$ of the incident wave is to be determined. For a solution of (2),(3),(4) the transmittance is the ratio between the time-averaged Poynting vectors in the respective media of incident and output regions. This is the ratio between incident and transmitted power for time-harmonic electromagnetic fields

$$
T=\frac{P_{\text {out }}}{P_{\text {in }}}=\frac{\frac{1}{2} n_{\text {out }}|E(L)|^{2}}{\frac{1}{2} n_{\text {in }}\left|E_{\text {inc }}(0)\right|^{2}} .
$$

In this context, a transmission resonance can be defined as a local maximum of the transmittance in a selected frequency region of otherwise low transmittance.

Alternatively, a finite structure can be viewed as an open system with transparent boundaries which permit leakage of energy to the exterior. Just as before, the behavior of the electric field in the interior $x \in(0, L)$ is governed by the Helmholtz equation

$$
\partial_{x}^{2} Q(x)+k^{2}(x) Q(x)=0
$$

now with outgoing wave boundary conditions

$$
\begin{gathered}
\left(\partial_{x} Q(x)+i k_{i n} Q(x)\right)_{x=0}=0 \\
\left(\partial_{x} Q(x)-i k_{\text {out }} Q(x)\right)_{x=L}=0 .
\end{gathered}
$$


This constitutes an eigenvalue problem for the frequency $\omega$ as the complex eigenvalue and the electric field $Q(x)$ as eigenfunction, called Quasi-Normal Mode. QNMs appear as discrete infinitely countable set of solutions of this eigenvalue problem [6]. They are unbounded functions that blow-up for $x \rightarrow \pm \infty$, so they are essentially different from resonant field solutions of the transmittance problem.

A finite, but internally periodic structure, i.e. a finite multilayer grating possesses a QNM spectrum that appears to be related to the bandgap structure and resonance properties of the transmission / reflection spectra. Representations in terms of QNMs for finite, periodic structures have been investigated in [9-11]. The positions of complex eigenfrequencies in the complex plane are arranged in such way that suggest the presence of bandgap regions in the transmittance response. Occurrence of the bandgap is to be expected for slices in the complex frequency plane where eigenvalues are not present. The edge of the bandgap in these terms can be estimated by taking real parts of the eigenfrequencies at the ends of separated arranged sets of eigenvalues [9-11]. The introduction of a defect in an otherwise periodic multilayer results in isolated QNMs with the real parts of their complex eigenfrequencies inside the bandgap region, as shown in section 3 .

In $[9,11]$ it has been noticed that the squared modulus of a QNM with complex frequency $\omega_{p}$ is similar to the field intensity inside the structure for a real frequency $\omega \approx \operatorname{Re}\left(\omega_{p}\right)$. This is a good approximation in particular for high-Q transmission resonances and for QNMs with eigenfrequencies with small imaginary parts. Still, a proper approximation of the field (not intensity) in the transmission problem requires many terms in an expansion based solely on QNMs.

\subsection{Solutions by transfer-matrix method}

We consider multilayer structures with piecewise constant refractive index distribution inside the finite spatial domain. Method for solving the transmittance (and eigenvalue) problems is the well known transfer matrix method (TMM) [4]. Solutions of the Helmholtz equation are given as combinations of left- and righttravelling waves in the $j$-th layer

$$
E_{j}(x)=A_{j} e^{i k_{j}\left(x-l_{j-1}\right)}+B_{j} e^{-i k_{j}\left(x-l_{j-1}\right)}
$$

for $x \in\left[l_{j-1}, l_{j}\right]$ in a region of constant index $n_{j}$ where $k_{j}=n_{j} \omega / c$ is the wave number in this layer. To connect the fields inside all layers we impose continuity conditions at the interfaces between consecutive layers,

$$
E_{j}\left(l_{j}\right)=E_{j+1}\left(l_{j}\right)
$$

and

$$
\partial_{x} E_{j}\left(l_{j}\right)=\partial_{x} E_{j+1}\left(l_{j}\right)
$$


These conditions lead to a system of equations that relate amplitudes of left- and right- traveling waves in different layers. They can be represented in matrix form. Ordered multiplication of the relevant matrices connects amplitudes in each layer of the structure, as well as the amplitudes in the incidence and output regions:

$$
\left(\begin{array}{c}
A_{\text {in }} \\
B_{\text {in }}
\end{array}\right)=\left(\begin{array}{ll}
m_{11}(\omega) & m_{12}(\omega) \\
m_{21}(\omega) & m_{22}(\omega)
\end{array}\right)\left(\begin{array}{c}
A_{\text {out }} \\
B_{\text {out }}
\end{array}\right) .
$$

The transmittance problem with incoming wave from the left is solved with $B_{\text {out }}=$ 0 for specified $A_{\text {in }}$ (amplitude of the incoming wave) with given real frequency $\omega \in R$. The amplitude transmission and reflection coefficients are expressed as

$$
\begin{aligned}
& t(\omega)=\frac{A_{\text {out }}}{A_{\text {in }}}, \\
& r(\omega)=\frac{B_{\text {in }}}{A_{\text {in }}} .
\end{aligned}
$$

If we choose conditions $A_{\text {in }}=B_{\text {out }}=0$, i.e. restrict the exterior solutions to purely outgoing waves, the eigenvalue problem with outgoing wave boundary conditions is addressed. With these conditions the system of equations can be nontrivially satisfied if

$$
m_{11}(\omega)=0 .
$$

Analytic continuation of transfer matrix into the complex plane enables us to find solutions of (15) as complex eigenvalues $\omega$ [24]. By substituting the eigenvalue into the field representation (9) we obtain the corresponding eigenfunction, up to a complex constant. To solve (15) we apply a numerical iteration procedure of Newton type. In cases when that method fails to converge due to closely spaced eigenvalues, we use a more powerful technique for determining complex solutions, based on the argument principle method from complex analysis [22].

\subsection{Field template and variational formulation for transmittance problem}

We specialize to finite periodic structures that possess transmission properties with a bandgap, i.e. with a region of frequencies of very low transmission. Introduction of suitable defects leads to a resonant transmission response inside the bandgap of the underlying periodic structure. We choose a field template for the transmittance problem as

$$
E(x, \omega) \simeq E_{m f}(x, \omega)+\sum_{p=1}^{N} a_{p}(\omega) Q_{p}(x),
$$

where $p$ is an index counting $N$ relevant QNMs . We take the relevant QNMs as those with the real part of their complex frequency in the given frequency range. 
We show, in terms of the successful application of the template (16), that the transmission resonance associated with the defect, appearing inside the bandgap, is triggered by the "mirror" field $E_{m f}$ of the periodic structure without defect, which for frequencies inside the bandgap is an almost completely reflected wave with only a weak tail that extends into the interior of the structure.

Note that $E_{m f}$ satisfies correct boundary conditions of the form (3) for the transmittance problem, while a superposition of QNMs can not cover the contribution of the incoming wave directly. The incoming field has to be included by other means when expansion into the complete set of QNMs is considered [11]. The inclusion of $E_{m f}$ (or some similar object) is essential in our approach as a means to represent the incoming wave. The mirror field does not extend far into the region of the defect where the contributions of the relevant QNMs are expected to be dominant.

Hence, according to template (16) the forced resonance response of the structure appears because the incident wave possesses a real frequency close to the real part of the complex eigenfrequency of a suitable QNM supported by the defect structure.

Obviously (16) constitutes only an approximate model for the transmittance problem in specific frequency regions, since neither $E_{m f}$ nor $Q$ satisfy all of equations (2)-(4). The residuals can be viewed as contributions from other QNMs in the complete set supported [13] by the defect structure, that are not included in (16). We shall see, however, that the template (16) leads to excellent approximations for the configurations of section 3 .

To determine the decomposition coefficients $a_{p}$ in our field template we employ a variational form of the transmittance problem. The transmittance problem corresponds to the equation and natural boundary conditions, arising from the condition of stationarity of the functional [25]:

$$
\begin{aligned}
L(E) & =\int_{0}^{L} \frac{1}{2}\left(\left(\partial_{x} E(x)\right)^{2}-k^{2}(x) E^{2}(x)\right) d x \\
& -\frac{1}{2} i k_{\text {in }} E^{2}(0)-\frac{1}{2} i k_{\text {out }} E^{2}(L)+2 i k_{\text {in }} A_{\text {inc }} E(0) .
\end{aligned}
$$

If $L$ becomes stationary, i.e. if the first variation of $L(E)$ vanishes for arbitrary variations of $E$, then $E$ satisfies (2), (3), and (4) as natural boundary conditions ${ }^{1}$.

Restricting the functional (17) to our field template (16), $L$ becomes a function of

\footnotetext{
1 The functional obtained from (17) by setting $A_{i n c}=0$, is formally related to the bilinear form (inner product), in which QNMs are orthogonal [6]. However, the frequency plays a different role in both cases: while in the functional (17) it is a given parameter, the bilinear form of [6] operates on objects that include the eigenfrequencies as arguments.
} 
the coefficients $a_{p}$, for given $E_{m f}$ and $Q_{p}$. The stationarity conditions then read:

$$
\frac{\partial L\left(a_{1}, a_{2}, \ldots, a_{N}\right)}{\partial a_{q}}=0, q=1, \ldots, N
$$

The optimal coefficients can be obtained as solutions of a system of linear equations

$$
\mathbf{A} \cdot \mathbf{a}=-\mathbf{b}
$$

where $\mathbf{a}=\left[a_{1}, a_{2}, \ldots, a_{N}\right]^{T}$ is the vector of coefficients to be determined. The components of the matrix $\mathbf{A}=\left[A_{q p}\right]_{N x N}$ and vector $\mathbf{b}=\left[b_{1}, . ., b_{N}\right]^{T}$ are

$$
\begin{aligned}
A_{q p} & =\int_{0}^{L}\left(\partial_{x} Q_{q} \partial_{x} Q_{p}-\frac{n^{2}(x) \omega^{2}}{c^{2}} Q_{q} Q_{p}\right) d x \\
& +i \frac{\omega}{c}\left(n_{\text {in }} Q_{q}(0) Q_{p}(0)+n_{\text {out }} Q_{q}(L) Q_{p}(L)\right)
\end{aligned}
$$

and

$$
\begin{aligned}
b_{q} & =\int_{0}^{L}\left(\partial_{x} E_{m f} \partial_{x} Q_{q}-\frac{n^{2}(x) \omega^{2}}{c^{2}} E_{m f} Q_{q}\right) d x \\
& +i \frac{\left(\omega_{q}-\omega\right)}{c}\left(n_{\text {in }} E_{m f}(0) Q_{q}(0)+n_{\text {out }} E_{m f}(L) Q_{q}(L)\right)+2 i n_{\text {out }} \frac{\omega}{c} A_{\text {inc }} Q_{q}(0) .
\end{aligned}
$$

By solving the system of equations (19) for each value of the real frequency $\omega$ the decomposition coefficients in the field representation for the transmittance problem are obtained. This enables approximation of the spectral transmittance and reflectance and the related field profile. The transmittance reads:

$$
T(\omega)=\frac{n_{\text {out }}}{n_{\text {in }}} t t^{*}=\frac{n_{\text {out }}}{n_{\text {in }}}\left|\frac{E_{m f}(L)+\sum_{p=1}^{N} a_{p}(\omega) Q_{p}(L)}{A_{\text {inc }}}\right|^{2} \text {. }
$$

The field in the region of incidence can be seen as a superposition of incident and reflected waves

$$
E(x)=A_{i n c} e^{i k_{i n} x}+r A_{i n c} e^{-i k_{i n} x},
$$

where $r$ is the amplitude reflection coefficient, related to the reflectance (power reflection defined as the ratio between the Poynting vectors of reflected and incident waves)

$$
R=r r^{*}=\left|\frac{E_{m f}(0)+\sum_{p=1}^{M} a_{p}(\omega) Q_{p}(0)}{A_{\text {inc }}}-1\right|^{2} .
$$




\section{Results and Discussion}

We specialize to structures with piecewise constant refractive index distribution of high $n_{H}$ and low refractive index $n_{L}$ layers, with quarter-wavelength optical thicknesses $L_{H}, L_{L}$ at a target frequency $\omega_{0}$. High index layers are denoted by $H$, low-index layers by $L$ and defect layers by $D$. Thus a finite symmetric periodic structure is represented by $(H L)^{N} H$, where $N$ is the number of the layer pairs. The defects are introduced as changes of the thickness of specific layers, but the method can handle defects introduced as changes of refractive indices of certain layers as well. In examples below we use only 1,2 and 3 most relevant QNMs corresponding to single-, double- and triple-cavity structures.

When single high-Q resonances inside the bandgap are considered, decomposition coefficients depend weakly on the frequency, apart from the Lorentzian approximation (eqns. $(28,29)$ ). Then the transmittance profile can be obtained analytically for the major part of the bandgap region around resonance position. If the full frequency dependence is included in $E_{m f}$ as described so far, then computational cost is comparable to direct TMM computations for the full structure, but captures adequately the deviation from the Lorentzian approximation. Further, we earn a certain degree of interpretability by being able to observe the interplay between the QNM basis modes.

\subsection{Symmetric single cavity structure}

We consider a layer arrangement $(H L)^{4} D(L H)^{4}$, as an example of a single cavity, with $n_{H}=3.42 n_{L}=1.45$, enclosed within two semiinfinite media of the same refractive index $n_{\text {in }}=n_{\text {out }}=1.0$. The defect is introduced as a change of the thickness $L_{H}$ in the central layer with high refractive index $n_{H}$ with $L_{D}=2 L_{H}$. The QNM spectrum for the original periodic structure and the structure with the defect is depicted in Fig. 2A). The QNM frequencies clearly show an arrangement in the complex plane, that reflects the presence of the bandgap in the transmittance response presented in Fig. 2B). For the defect structure a complex frequency in the QNM spectrum appears in the position of the transmission resonance in the bandgap (see Fig. 2A). The field profile of the transmission resonance and the profile of the QNM corresponding to the defect structure have similar pattern as depicted in Figs. 2 C) and D). The difference between the QNM and transmission resonance field is clearly visible in Fig. 2E), arising from different boundary conditions (around $x=0$, the transmittance field represents inwards travelling wave and QNM outwards travelling wave), consequently leading to entirely different behavior in the region where the incident wave is present. We take the mirror field in the template as the solution of the transmittance problem of the structure without defect at each frequency in the considered bandgap region. Approximating both the 
mode structure and the correct boundary conditions for the field representation of the TRM (transmission resonance mode Fig. 2C ), using the QNMs and the mirror field in Fig. 2F) leads to an excellent agreement between the approximation obtained form the field template and the exact TMM solution, as can be seen in Fig. 2G). An acceptable agreement between the approximated field profile and the TMM reference (exact solution) is valid in the whole bandgap frequency range. The field template including an exciting field, together with the variational procedure, provides a constructive quantitative way to relate QNMs and TRMs.
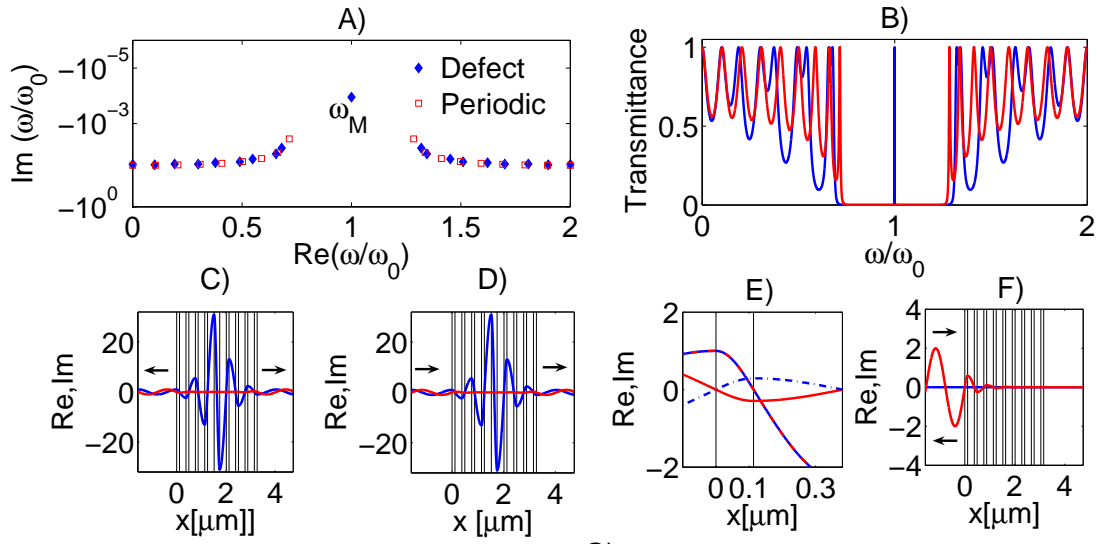

G)

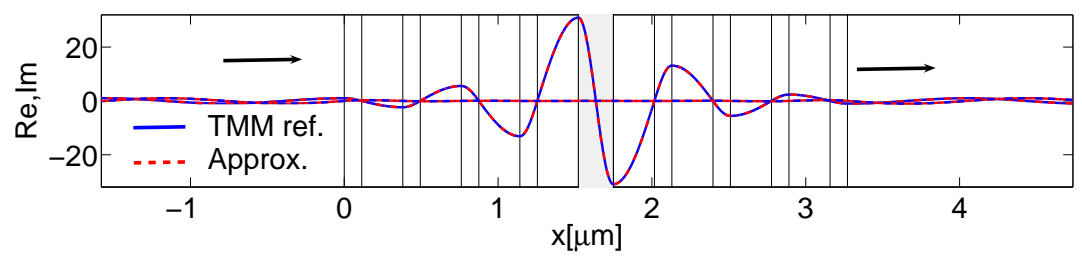

Fig. 2. A) Complex frequencies (eigenvalues) for periodic and single cavity structure B) Transmittance for periodic (dashed) and single cavity structure (continuous) C) Field pattern for a (defect) frequency at the center of the bandgap, real and imaginary parts D) Quasi normal mode corresponding to the complex eigenfrequency $\omega_{M}$ E) Comparison of the QNM for $\omega_{M}$ (solid line) and the transmission (defect) field (dotted line) in the region around $x=0$ where the incoming field is present. F) Mirror field for the (periodic) structure without defect for $\omega=\operatorname{Re}\left(\omega_{M}\right)$ G) Field associated with the transmission resonance in the defect structure obtained via variational approximation based on mirror field and relevant QNM and compared with TMM reference.

The transmittance (22) is compared with the TMM reference calculation, as shown in Fig. 3A). We observe an excellent agreement between the approximation and the TMM calculations. Fig. 3B) shows the frequency dependence of the decomposition coefficient $a_{M}$ for this structure. The resonant response is clearly reflected in this dependence, showing that the transmission resonance is connected with the excitation of the internal dynamics represented by the relevant QNM.

A common assumption made in the literature is that the spectral transmission for the single resonance situation, as described, is of a Lorentzian lineshape. Our method can analytically justify this assumption. We consider the contribution of a single 


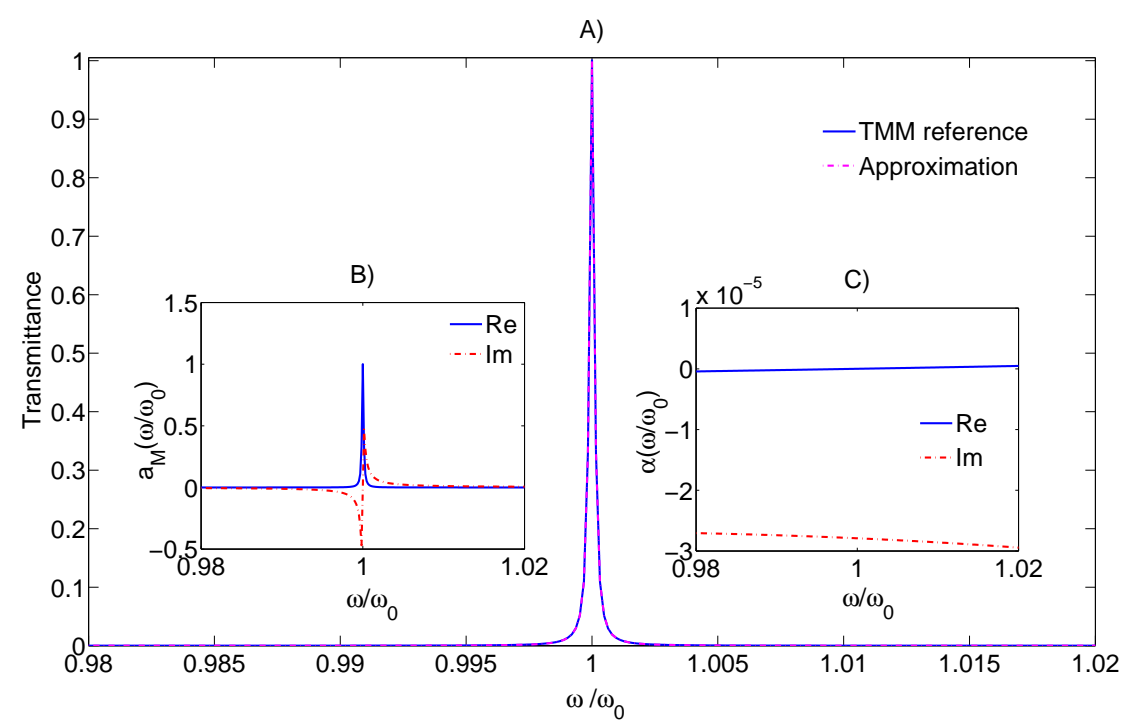

Fig. 3. A) Transmittance obtained from field representation using QNMs and TMM reference B) Decomposition coefficients C) Non-resonant part of the decomposition coefficient.

QNM in the field template (16). The equation for the decomposition coefficient then reads

$$
A a(\omega)+b=0 .
$$

After partial integration (21) can be given the form

$$
b=\omega^{2} \int_{0}^{L} \frac{n_{0}^{2}(x)-n^{2}(x)}{c^{2}} E_{m f} Q_{q} d x
$$

with $n_{0}$ being the refractive index distribution for the finite periodic (unperturbed) structure. After partial integration, (20) reads

$$
A=\left(\omega_{q}^{2}-\omega^{2}\right) \int_{0}^{L} \frac{n^{2}(x)}{c^{2}} Q_{q}^{2} d x+i \frac{\left(\omega_{q}-\omega\right)}{c}\left(n_{\text {in }} Q_{q}^{2}(0)+n_{\text {out }} Q_{q}^{2}(L)\right) .
$$

The amplitude transmission coefficient can be approximated as

$$
t(\omega)=\frac{E_{m f}(L)+a(\omega) Q_{q}(L)}{A_{\text {inc }}} \simeq \frac{a(\omega) Q_{q}(L)}{A_{\text {inc }}}
$$

assuming that the periodic structure represents a "good mirror" with the property $E_{m f}(L) \simeq 0$. The frequency dependence of the transmission amplitude then comes from the decomposition coefficient

$$
a(\omega)=\frac{\alpha(\omega)}{\omega-\omega_{q}} \simeq \frac{\alpha}{\omega-\omega_{q}},
$$


where

$$
\alpha(\omega)=\frac{\omega^{2} \int_{0}^{L} \frac{n_{0}^{2}(x)-n^{2}(x)}{c^{2}} E_{m f} Q_{q} d x}{\left(\omega+\omega_{q}\right) \int_{0}^{L} \frac{n^{2}(x)}{c^{2}} Q_{q}^{2} d x+\frac{i}{c}\left(n_{\text {in }} Q_{q}^{2}(0)+n_{\text {out }} Q_{q}^{2}(L)\right)} .
$$

Term (29) is non-resonant in character; in the case of a very narrow resonance $\omega \simeq \operatorname{Re}\left(\omega_{q}\right)$ and $\operatorname{Im}\left(\omega_{q}\right) \ll \operatorname{Re}(\omega)$ can be shown to depend weakly on the frequency, see Fig. 3C). Therefore, in the framework of our approximate model equation (28) represents the Lorentzian like shape as shown in Fig 3B). This result agrees with those obtained previously in literature $[9,11,20,7]$ Here it follows from a completely different approach and further supports the conclusion that our method adequately captures the resonance character of the transmission.

\subsection{Asymmetric single cavity}

Now the internal structure of the previous example is enclosed within two semiinfinite media of different refractive indices $n_{\text {in }}=1$ and $n_{\text {out }}=5$ (as a somewhat artificial example to emphasize asymmetric nature of the structure). The QNM spectrum and transmittance shown in Fig. 4A) and 4B) and the QNM profile in Fig. 4C) suggest that the difference between the symmetric and asymmetric structures is reflected in the shift of frequency positions in the complex plane. The same qualitative behavior can be seen as in Fig. 2A) and 2B), i.e. a single resonance appears in the bandgap region when the defect is introduced, now with the lower transmittance level (corresponding to the reflection at an interface between media with indices $n_{\text {in }}$ and $n_{\text {out }}$ [4]). A similar field template as for the symmetric structure is used. This choice is further confirmed by the excellent agreement between the approximation of the transmittance with the TMM reference calculation shown in Fig. 4D).

\subsection{Double cavity structure}

For this example, we consider a layer arrangement $(H L)^{4} D(L H)^{2} L D(L H)^{4}$, where two defects are introduced as changes of thicknesses of layers $L_{D}=2 L_{H}$, where $n_{D}=n_{H}$. The refractive index outside the structure is the same on both sides. The values of the refractive indices are the same as in section 3.1. These defects are forming two FP (Fabry-Perot like) resonant cavities enclosed by two identical mirrors and one separating mirror. The resonant response of the double-cavity structure is represented by two complex frequencies in the bandgap region as shown in Fig. 5A). The corresponding transmittance plot shows two distinct transmission resonances in the bandgap region Fig. 5B). The QNMs for these two defect-induced 

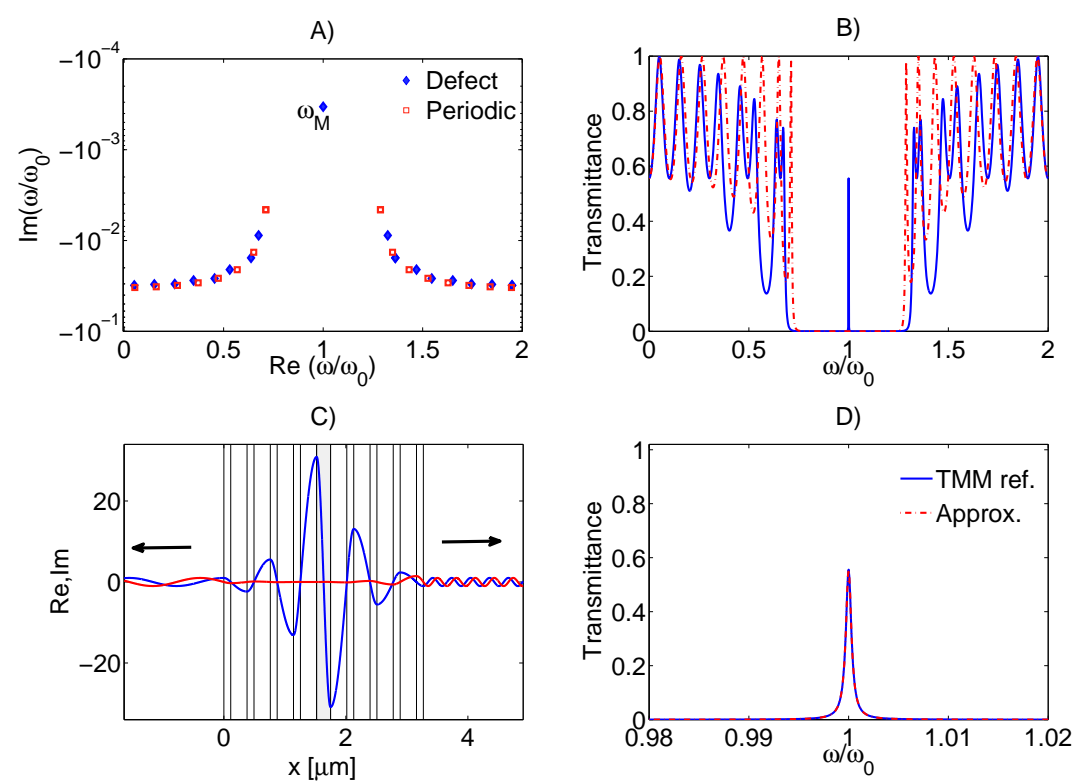

Fig. 4. A) QNM spectrum and B) Transmittance for the asymmetric periodic (dashed) and defect (continuous) structure C) QNM for the defect structure D) Transmittance obtained from the field representation using QNMs and the TMM reference.

eigenfrequencies are shown in Fig 6C) and 6D). Symmetric and skew-symmetric behavior of the eigenfields is present, arising form the overall symmetry of the structure.
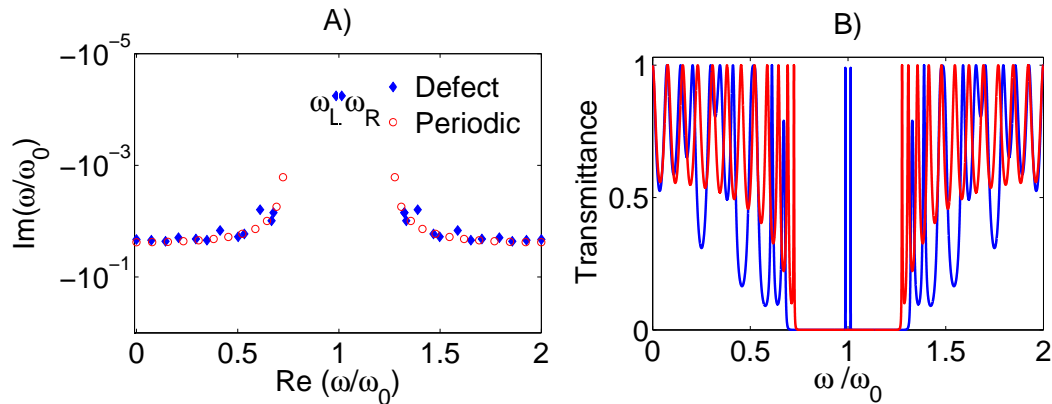

C)

D)
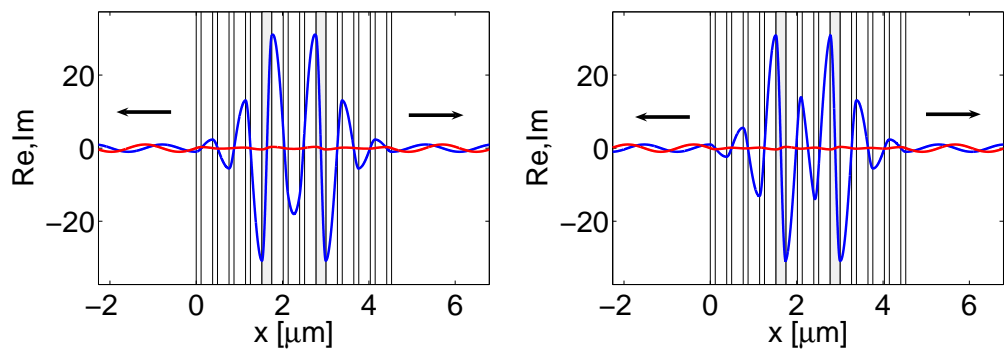

Fig. 5. A) QNM spectrum B) Transmittance for periodic and double cavity structure; QNMs corresponding to complex frequencies in the bandgap region C) QNM for $\omega_{L}$ D) QNM for $\omega_{R}$ 
Fig. 6A) and 6B) shows the decomposition coefficients and the approximated transmittance response that is in excellent agreement with TMM reference. The field template based on the mirror field of the structure without defects and linear combination of the two relevant QNMs enables an excellent field representation of the transmission resonance modes as can be seen from Figs. 6C) and 6D).
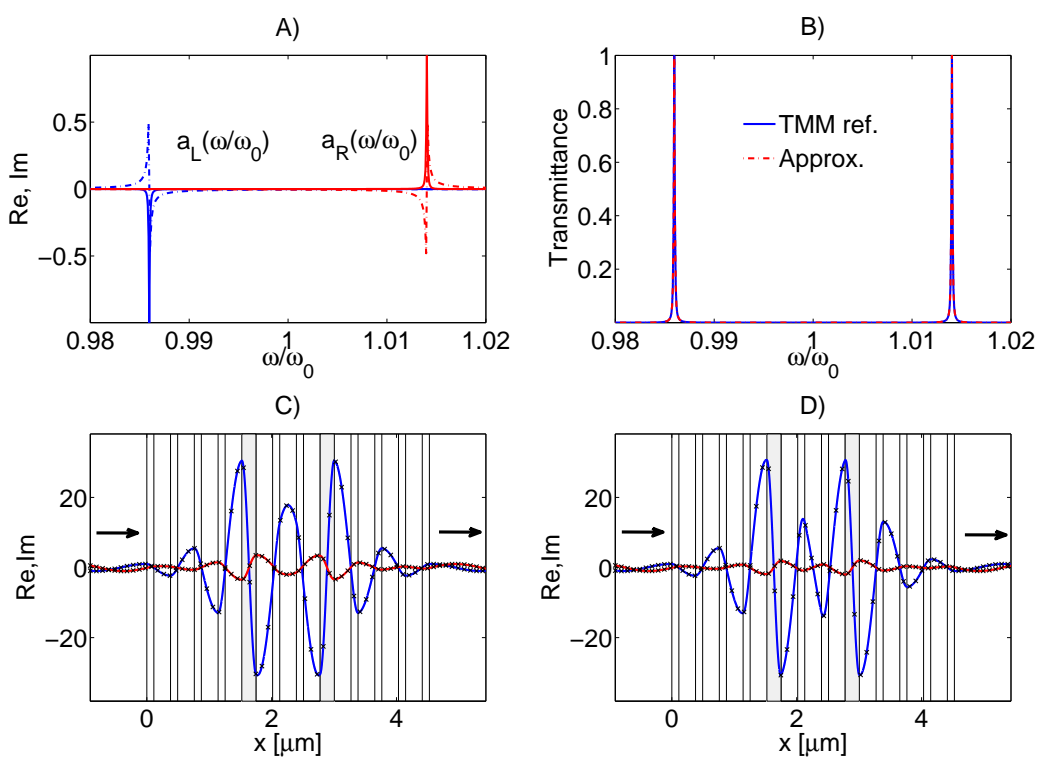

Fig. 6. A) Decomposition coefficients. B) Transmittance obtained from the field representation using QNMs (dashed) and TMM reference (continuous). C) and D): approximated field obtained from the field representation using QNMs (marker) and TMM reference for the frequency of transmission resonance (solid line) for $\omega=\operatorname{Re}\left(\omega_{L}\right)$ and) $\omega=\operatorname{Re}\left(\omega_{R}\right)$.

This example can be considered as a case of strongly coupled FP cavities where the interaction is sufficient to introduce a significant separation of the resonance frequencies. This is reflected in the positions of the defect QNM eigenfrequencies of the defect structure. Our approximation method enables both an accurate field representation and predicts the proper resonant transmission.

\subsection{Multiple cavity structure with flat-top narrow-band transmission}

As last example we choose an asymmetric triple cavity structure with layer arrangement coded as $(H L)^{4} L(H L)^{9} L(H L)^{9} L(H L)^{4}, n_{H}=2.1, n_{L}=1.45, n_{\text {in }}=$ $n_{\text {out }}=1.52, L_{H}, L_{L}$-quarter-wavelength [26]. This structure introduces three complex eigenfrequencies in the bandgap region, as shown in Fig. 7A). The important feature is that it provides a narrow-band flat-top transmission inside the bandgap region as can be seen in Fig 7B). The closely spaced eigenfrequencies and the 
corresponding QNMs are shown in Fig. 8. The proximity of the complex frequencies reflects weak coupling between the three individual FP cavities formed by the defects. Fig. 9 shows the decomposition coefficients and the approximated transmittance (compared with the TMM reference). The close proximity of the eigenfrequencies is reflected in the substantial overlapping of the frequency regions where all three decomposition coefficients contribute. The field pattern in this region is clearly produced by the combination of three relevant QNMs. Obviously all three QNMs play a significant role over the whole transmission band. The approximated field profiles for the transmission pass-band and the immediate bandgap region agree well with the TMM reference, as shown in Fig. 10.

We wish to point out that direct TMM calculations are not suitable for estimating the resonance origin of the transmission band.Also, some of the methods used in literature, that estimate complex eigenfrequencies by matching the transmittance spectrum to the Lorentzian lineshape functions and estimate eigenfields through association of the TRMs with the QNMs, see [27] and references therein, are not efficient in this case. Neither can estimates of the complex eigenfrequencies and QNMs based on FDTD (finite difference time domain) simulations deal easily with this type of structures with flat-top transmission, see [2] and references therein. In contrast our model permits to observe directly the relevance of the individual QNMs at different frequencies (i.e. the magnitude of the expansion coefficients $\left.a_{p}\right)$.
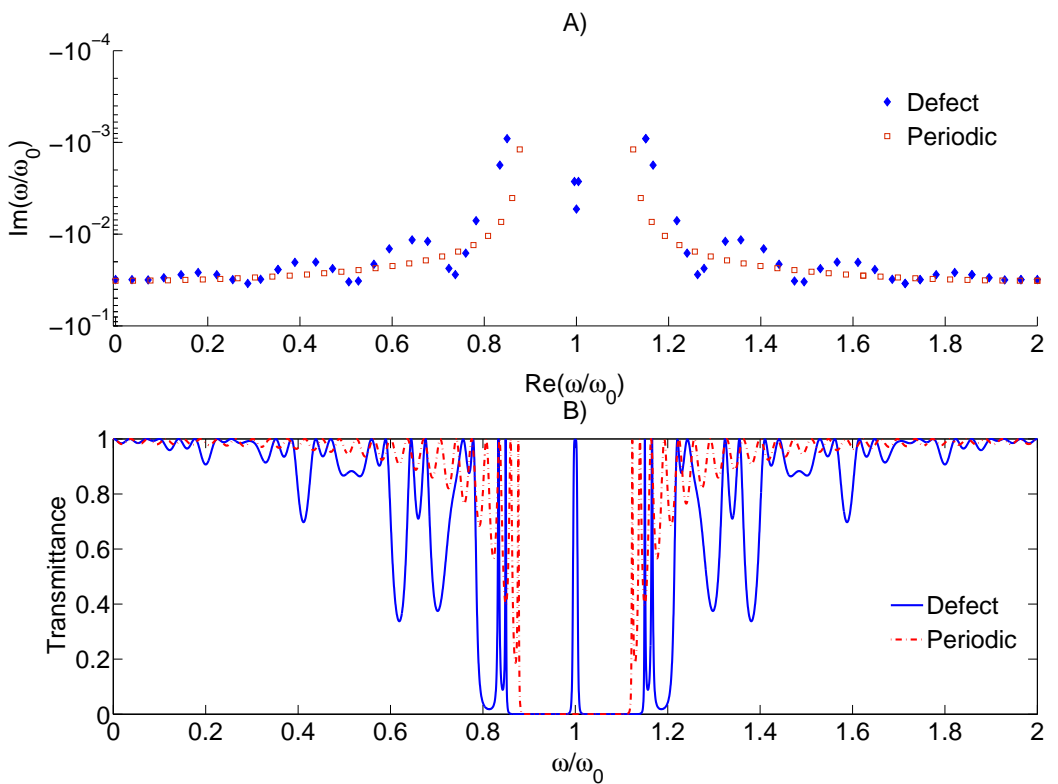

Fig. 7. QNM eigenfrequencies A) for the triple-defect structure and periodic (unperturbed) structure, and transmittance B). 


\section{Conclusions}

We proposed a constructive way of connecting a quasi-normal mode (eigenmode) description with transmission resonance properties for optical defect microcavities in 1D multilayer structures. The approach is meant specifically for approximations of the defect induced transmission modes existing in the bandgap of otherwise periodical structures.

The field representation using a mirror field and the most relevant QNMs enables very accurate field representations for field profiles in the transmittance problem. The approximated spectral transmittance agrees excellently with the TMM reference. We emphasize the open and finite nature of the structures by directly characterizing resonance properties via an investigation of the quasi-normal mode spectrum.

Numerical examples suggest that the method is valid for single and multiple cavity structures in both symmetric and nonsymmetric layer arrangements and both weak and strong couplings between defects. Moreover, our method allows to examine directly the resonance nature of the transmission response in cases where it is very hard to establish this from exact solutions of the transmission problem, such as provided by the TMM method.

The approach quantifies directly the physical viewpoint, where the defect cavities are regarded as externally forced oscillators. The field representations obtained using QNMs have a better foundation in the physics and nature of the realistic, finite structures, when compared with methods that assumes periodic boundary conditions for the structure.

We believe that our approach can be generalized to 2D and 3D structures as an acceptable characterization for both fields and response functions. Provided that suitable QNM basis fields can be made available by analytical or numerical means, generalizations could be based on the functional representations of the frequency domain Maxwell equations for higher dimensions [28],[29].

4.0.0.1 Acknowledgment This work is financially supported by NanoNed, flagship NanoPhotonics, project TOE.7143.

\section{References}

[1] J. D. Joannopoulos. Photonic Crystals: Molding the Flow of Light. Princeton University Press, 1995. 
[2] J.-M Lourtioz. Photonic Crystals: Towards Nanoscale Photonic Devices. Springer, 2005.

[3] K. Sakoda. Optical Properties Of Photonic Crystals. Springer, 2004.

[4] P. Yeh. Optical waves in layered media. Willey, 1988.

[5] E. S. C. Ching, P. T. Leung, and K. Young. Optical processes in microcavities-the role of quasi-normal modes. In R.K.Chang and A.J.Campillo, editors, Optical processes in mirocavities, chapter 1, pages 1-77. World Scientific, 1996.

[6] E. S. C. Ching, P. T. Leung, A. Maassen van den Brink, W. M. Suen, S. S. Tong, and K. Young. Quasi-normal mode expansion for waves in open systems. Rev. Mod. Phys, 70(4):1545-1554, 1998.

[7] A. Sopahaluwakan. Characterization and simulation of localized states in periodic strucures, PhD Thesis. University of Twente, 2006.

[8] S. Severini, A. Settimi, C. Sibilia, M. Bertolotti, A. Napoli, and A. Messina. Quasinormal frequencies in open cavities: An application to photonic crystals. Acta Phys. Hung. B, 23/3-4:135-142, 2005.

[9] A. Settimi, S. Severini, N. Mattiucci, C. Sibilia, M. Centini, G. D'Aguanno, M. Bertolotti, M. Scalora, M. Bloemer, and C. M. Bowden. Quasi-normal mode description of waves in one-dimensional photonic crystals. Phys. Rev E, 68:026614/1026614/11, 2003.

[10] S. Severini, A. Settimi, C. Sibilia, M. Bertolotti, A. Napoli, and A. Messina. Second quantization and atomic spontaneous emission inside one-dimensional photonic crystals via a quasi-normal mode approach. Phys. Rev. E, 70:056614-1-11, 2004.

[11] M. Bertolotti. Linear one-dimensional resonant cavities. In F.Michelotti, A.Driessen, and M.Bertolotti, editors, Microresonators as building blocks for VLSI photonics, volume 709 of AIP Conference Proceedings, pages 19-47. AIP, 2004.

[12] S. Severini, A. Settimi, C. Sibilia, M. Bertolotti, A. Napoli, and A. Messina. Quantum counter-propagation in open optical cavities via the quasi-normal-mode approach. $J$. Laser Phys., 16/6:911-920, 2006.

[13] P. T. Leung, S. Y. Liu, and K. Young. Completeness and orthogonality of quasinormal modes in leaky optical cavities. Phys. Rev. A, 49(4):3057-3067, 1994.

[14] P. T. Leung, S. S. Tong, and K. Young. Two-component eigenfunction expansion for open systems described by wave equation i: completeness of expansion. J.Phys. A: Math.Gen., 30:2139-2151, 1997.

[15] P. T. Leung, S. S. Tong, and K. Young. Two-component eigenfunction expansion for open systems described by wave equation ii: linear space structure. J.Phys. A: Math.Gen., 30:2153-2162, 1997.

[16] P. T. Leung, W. M. Suen, C. P. Sun, and K. Young. Waves in open systems via a biorthogonal basis. Phys. Rev. E, 57(5):6101-6104, 1998. 
[17] Alec Maassen van den Brink and K. Young. Jordan blocks and generalized biorthogonal bases: realizations in open wave systems. J. Phys. A: Math. Gen., 34:2607$2624,2001$.

[18] B. Hoenders. On the completeness of the natural modes for quantum mechanical potential scattering. J.Mat.Phys., 20:329-335, 1979.

[19] B. Hoenders and M. Bertolotti. The quasi normal mode description of the scattering process by dispersive photonic crystals. Proc. SPIE, 6182:61821F1-9, 2006.

[20] K. C. Ho, P. T. Leung, Alec Maassen van den Brink, and K. Young. Second quantization of open systems using quasi normal modes. Phys. Rev. E, 58(3):29652978, 1998.

[21] S. M. Dutra and G. Nienhuis. Quantized mode of a leaky cavity. Phys. Rev. A, 62:063805-1-13, 2000.

[22] D. Felbacq and R. Smaâli. Density of states for finite photonic crystals. Phys. Rev. B, 67:085105, 2003.

[23] D. Felbacq. Numerical computation of resonance poles in scattering theory. Phys. Rev. E, 64:047702, 2001.

[24] R. G. Newton. Scattering theory of waves and particles. Dover publications, 2002.

[25] E. W. C. van Groesen and J. Molenaar. Advanced Modeling in Science. SIAM publishers, 2007.

[26] C. K. Madsen and J. H. Zhao. Optical filter design and analysis: A signal processong approach. John Willey Sons, 1999.

[27] E. Anemogiannis, E. N. Glytsis, and T. K. Gaylord. Determination of guided and leaky modes in lossless and lossy planar multilayer optical waveguides: Reflection pole method and wavevector density method. J. Light. Tech, 17(5):929-941, 1999.

[28] C. Vasallo. Optical waveguide concepts. Elsevier, 1991.

[29] M. Hammer. Hybrid analytical/numerical coupled-mode modeling of guided wave devices. J. of Light. Tech., 25(9):2287-2298, 2007. 

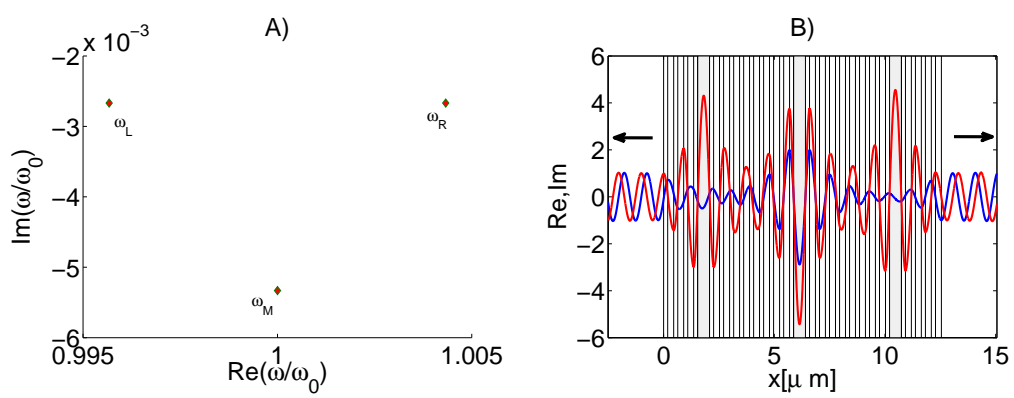

C)

D)
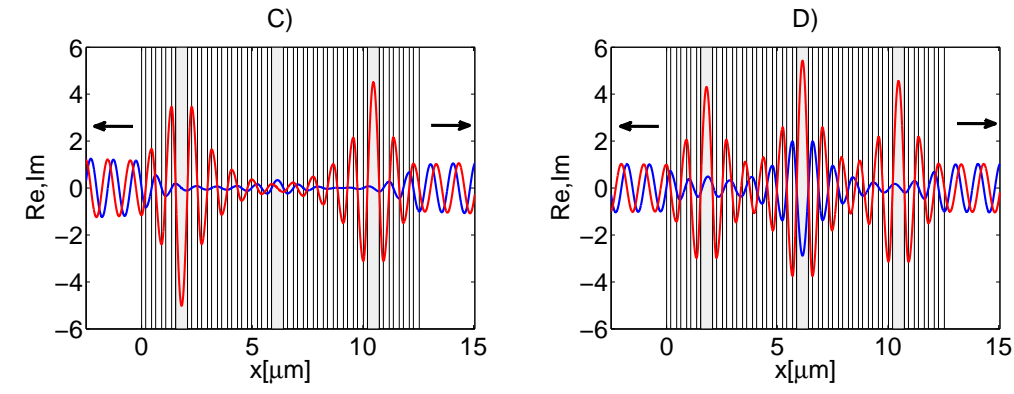

Fig. 8. A) Complex eigenfrequencies of defects induced QNMs in the triple-cavity structure, QNMs for $\omega_{L}$ B) $\omega_{M}$ C) and $\omega_{R}$ D).
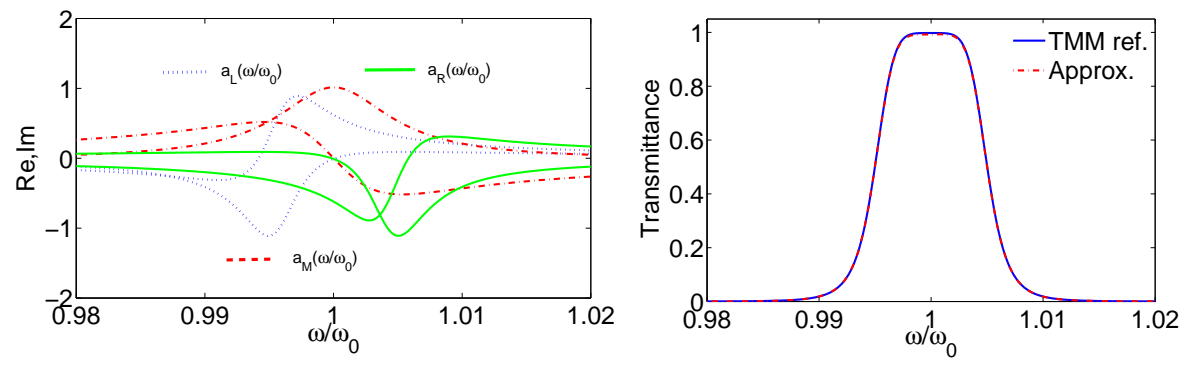

Fig. 9. A) Decomposition coefficients for the field representation, corresponding to the QNMs associated with $\omega_{L}, \omega_{M}, \omega_{R}$ B) Transmittance obtained from the field representation using QNMs and TMM reference 
A)

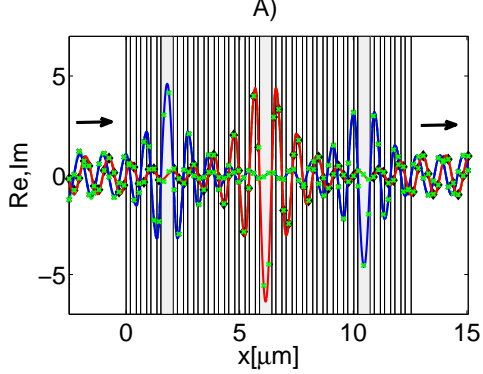

C)

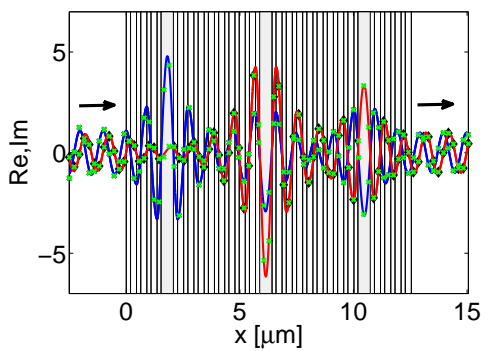

B)

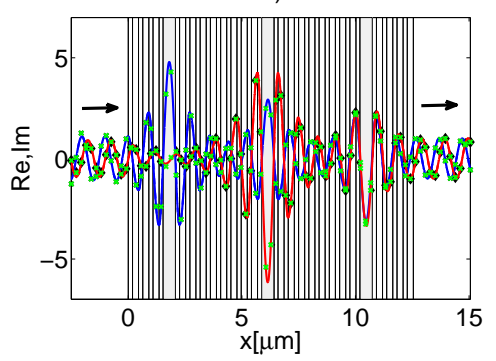

D)

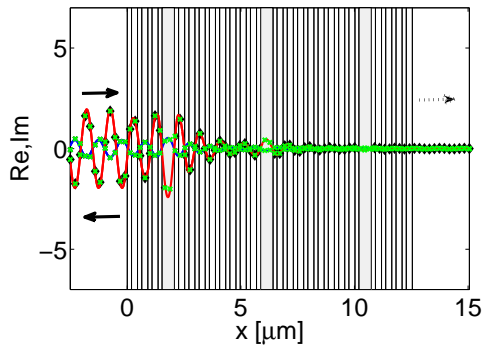

Fig. 10. Field profiles for transmission in and out of the pass-band obtained from the field representation using QNMs (marker) and compared to the TMM reference (solid line) A) $\omega / \omega_{0}=1$ B) $\omega / \omega_{0}=1.002$ C) $\omega / \omega_{0}=0.998$ D) $\omega / \omega_{0}=0.98$ 